\title{
Diseño de un modelo basado en criterios e indicadores de características para la evaluación de los sitios webs de archivos
}

\author{
Isabel SANZ CABALLERO \\ Junta de Extremadura. Consejería de Fomento. Archivo de Gestión \\ isancab@alcazaba.unex.es \\ Cristina FABA PÉREZ \\ Universidad de Extremadura. Departamento de Información y Documentación \\ cfabper@unex.es
}

Recibido: Septiembre 2011

Aceptado: Julio 2012

Resumen: Los archivos no pueden quedarse al margen de Internet puesto que la Red se ha convertido en una herramienta imprescindible para el desarrollo de las sociedades y para dar a conocer los servicios y los productos que ofrecen las instituciones. Esta apertura y difusión del archivo a través de la Web contribuye, sin duda, al incremento de su visibilidad. Ahora bien, el aumento de los archivos en Internet también ha traído consigo la necesidad de comprobar qué nivel de calidad presentan. Para ello, es preciso evaluarlos atendiendo a unos indicadores o características específicas relacionadas con su diseño, estructura o contenido. El objetivo principal del presente trabajo es el diseño de una plantilla exhaustiva de características específicas para la evaluación de los sitios web de archivos. Los resultados y las conclusiones del trabajo demuestran que es posible diseñar un modelo específico de características determinando cuáles son las que mejor representan la calidad de un archivo en la Web.

Palabras clave: Archivos; análisis de características específicas; calidad; modelo de evaluación sitios web.

\section{Design of a model-based criteria and characteristics for the evaluation of archives websites}

\begin{abstract}
Archives cannot be left out the Internet as the Web has become an indispensable tool for the development of the societies and to publicize the services and products that the institutions offer. This openness and diffusion of the archive through the Internet has also brought the need to check what level of quality they present. To do that it is necessary to evaluate them and to attend to some indicators or specific features related to their design, structure or contents. The main target of his work is to design a comprehensive template of specific characteristics for the evaluation of archive websites. The results and conclusions of this work demonstrate that it is possible to design a specific model of features determining which ones are those that best represent the quality of an archive on the Web.
\end{abstract}

Keywords: Archives; design of specific model; evaluation; quality; websites. 


\section{INTRODUCCIÓN}

La interacción informática ha cambiado espectacularmente el mundo en el que vivimos eliminando las barreras del tiempo y la distancia, y permitiendo a las personas e instituciones compartir información y trabajar en colaboración. El avance tecnológico continúa a un ritmo cada vez más rápido y las nuevas tecnologías aumentan la velocidad de transferencia de información, posibilitando tener al instante cualquier dato solicitado. En este entorno, donde el crecimiento de Internet es constante y exponencial, los archivos no pueden quedarse al margen puesto que la Red se ha convertido en una herramienta imprescindible para el desarrollo de las sociedades y para dar a conocer los servicios y los productos que ofrecen las instituciones.

Según la Ley 16/1985, de 25 de junio, del Patrimonio Histórico Español, los archivos se definen como "conjuntos orgánicos de documentos, o la reunión de varios de ellos, reunidos por las personas jurídicas, públicas o privadas, en el ejercicio de sus actividades, al servicio de su utilización para la investigación, la cultura, la información y la gestión administrativa. Asimismo, se entienden por archivos las instituciones culturales donde se reúnen, conservan, ordenan y difunden para los fines anteriormente mencionados dichos conjuntos orgánicos". Durante los últimos veinticinco años, los archivos han profundizado en la aplicación de las tecnologías de la información a un ritmo espectacular. Sin duda, ha influido notablemente la aparición de normativa internacional para la descripción archivística como ISAD (G) (General International Standard Archival Description, Norma Internacional General de Descripción Archivística) e ISAAR (CPF) (International Standard Archival Authority Record for Corporate Bodies, Persons, and Familias, Norma Internacional sobre los Registros de Autoridad de Archivos relativos a Instituciones, Personas y Familias) y para la transmisión de esta información en formatos estructurados como las definiciones de tipo de documento EAD (Encoded Archival Description, Descripción Archivística Codificada) y EAC (Encoded Archival Context, Contexto Archivístico Codificado). Igualmente, la importancia de la gestión archivística en cualquier institución puede apreciarse en la publicación de normas como la ISO 15489 Information and documentation records management (2001) o la ISO 23081 Information and documentation records management proceses metadata for records (2004).

Sin apartarse de las funciones tradicionales de conservadores de la memoria histórica, los archivos desempeñan un papel fundamental en todo lo relacionado con la gestión integral de cualquier tipo de información durante todo su ciclo de vida, desde su nacimiento hasta su expurgo o conservación permanente, convirtiéndose en piezas clave de las instituciones para las que trabajan. Esta consideración ha aumentado a lo largo de los años, a medida que las instituciones han ido difundiendo a través de la Web las ingentes cantidades de información contenidas en los documentos primarios que atesoran. Esta difusión, sin embargo, ha planteado problemas para su localización, búsqueda y recuperación en el 
entorno distribuido de la Web, haciendo necesaria la creación de un nuevo tipo de estructuras de información y aplicaciones informáticas que no sólo sean capaces de solucionar eficazmente los procesos archivísticos tradicionales, sino de añadir nuevas funcionalidades basadas fundamentalmente en el acceso y la difusión inteligente de la información, y haciendo posible crear/producir y no sólo conservar/consumir nuevos recursos electrónicos al servicio de todos los usuarios y del archivo mismo (Rodríguez Bravo, 2007). Esta apertura y difusión del archivo a través de la Web contribuye, sin duda, al incremento de su visibilidad para segmentos de usuarios que desconocen su existencia (Más Bleda, Sánchez Baena y Chaín Navarro, 2007).

Ahora bien, el incremento de los archivos en la Red, también ha traído consigo la necesidad de comprobar si dichos archivos están funcionando correctamente y qué nivel de calidad presentan. Para ello, es preciso evaluarlos atendiendo a unos parámetros o características relacionadas con su diseño, estructura o contenido. Estos parámetros forman parte de un conjunto de indicadores conocidos como "indicadores web(o)métricos de características" (Al-Duwairi, Faba Pérez y Vargas Quesada, 2010).

$\mathrm{Si}$ bien se han realizado algunas investigaciones para medir diversas características o indicadores web(o)métricos de los archivos en Internet (Cuadrado Fernández y Gómez Domínguez, 2002; Crespo, 2007; Kim y Lee, 2007; Chaín Navarro y Más Bleda, 2009), los modelos de características que se han empleado, en general, utilizan indicadores demasiado universales, es decir, se pueden aplicar en ámbitos muy heterogéneos, lo que implica valoraciones menos precisas en los resultados específicos de un área determinada. Para solventar esta necesidad, el objetivo principal del presente trabajo es el diseño de una plantilla exhaustiva de características para la evaluación específica de los sitios web de archivos, independientemente de su tipología, determinando cuáles son las características que mejor representan la calidad de un archivo en la Web. Se parte de la hipótesis de que es posible diseñar un modelo de características específicas para los archivos que permita obtener unos resultados objetivos sobre la calidad los mismos en la Web.

\section{DATOS Y METODOLOGÍA}

Para diseñar el modelo de características que permita la evaluación específica de los sitios web de archivos, se utiliza una metodología descriptiva que, partiendo del análisis de la bibliografía al respecto, permita desarrollar un modelo exhaustivo de características específico para el entorno de los archivos. Por consiguiente, los datos con los que se trabaja son los indicadores seleccionados en la bibliografía analizada y, especialmente, las nuevas características planteadas en el presente trabajo. La elección de los indicadores se justifica por la importancia asignada a los mismos en la bibliografía consultada y en el propio proceso 
archivístico, para lo que se han seguido las aportaciones a conceptos archivísticos fundamentales de autores como Schellemberg (1958), Lodolini (1993), Delgado Gómez y Cruz Mundet (2011), Heredia Herrera (2011) o Cruz Mundet (2011).

El análisis de características consiste en identificar las características de los sitios web que mejor satisfacen las necesidades de los usuarios que navegan por ellos. Se fundamenta en el hecho de que los sitios web por más disímiles que sean, tienen unas características comunes o indicadores de calidad que permiten su evaluación. Dichas características pueden ser subjetivas (recopiladas por el analista) y formales (recopiladas de forma automática, lo que justifica su objetividad) (Faba Pérez, Guerrero Bote y Moya Anegón, 2004).

Existen bastantes trabajos sobre análisis de características en el contexto de las unidades informativas. Sobre todo, se han realizado investigaciones en el campo de las bibliotecas, donde la mayoría de los trabajos se han centrado en el análisis de características subjetivas (Clausen, 1999; Falk, 1999; Joint, 2001; Osorio, 2001; Chao, 2002; Clyde, 2004; Novljan y Žumer, 2004), frente al análisis de indicadores formales (Stover y Zink, 1996; Craven, 2000; Spindler, 2002; Providenti, 2004; Schmetzke, 2005; Caballero Cortés, Faba Pérez y Moya Anegón, 2009).

En el caso de los archivos, a diferencia de las bibliotecas, existen menos investigaciones sobre análisis de sus características, ya que, aunque se pueden encontrar trabajos que relacionan los archivos con Internet y la World Wide Web (Martínez González, 2000; Gil García, 2001 y Torrado Morales, 2007), son escasos los que desarrollan modelos de características, si bien, algunos de ellos se pueden encontrar en los trabajos de Ruiz Pérez, Martín Fernández y Plasencia Rodríguez (1999), Cuadrado y Gómez (2002), Gil García (2003), Roba (2003), Crespo (2007), Kim y Lee (2007), Chaín Navarro y García González (2009) y Chaín Navarro y Más Bleda (2009), Más Bleda y Chaín Navarro (2009). De cualquier forma, estos últimos modelos utilizan, en general, indicadores demasiado universales que se pueden aplicar en ámbitos muy heterogéneos.

Por ello, en el presente trabajo, se diseña una plantilla de indicadores para la evaluación de sitios web de archivos donde se distinguen dos tipos de características:

- Características generales que debe contener un sitio web de archivos. En este caso, se han tomando como referencia los modelos universales de Hassan Montero y Martín Fernández (2003), Nielsen y Thair (2002) y Jiménez Piano (2001);

- Características especificas imprescindibles de un sitio web de archivos. En este caso, se han tomado como referencia los modelos previos sobre análisis de características en archivos de Ruiz Pérez, Martín Fernández y Plasencia Rodríguez (1999), Cuadrado y Gómez (2002), Gil García (2003), Roba (2003), Crespo (2007), Kim y Lee (2007), Chaín Navarro y García 
González (2009), Chaín Navarro y Más Bleda (2009) y Más Bleda y Chaín Navarro (2009); así como el marco teórico existente en la Archivística, para lo que se han consultado, tanto obras de relevancia en el área (Schellemberg, 1958; Lodolini, 1993; Cruz Mundet, 1994, 2011; Heredia Herrera, 1989, 2011; Conde Villaverde, 1992; Duplá del Moral, 1997; Fernández Gil, 1997; Delgado Gómez y Cruz Mundet, 2011), como trabajos especializados en indicadores de gestión en archivos (Sierra Escobar, 2012), y manuales de normas y procedimientos de numerosos archivos (Manual de Normas y Procedimientos del Archivo Universitario de Valladolid; Manual del Archivo General de la Universidad Carlos III de Madrid; Manual de Archivo de Oficina de la Junta de Castilla y León, etc.). Los indicadores de características específicas se han agrupado en sus criterios respectivos.

\section{RESULTADOS}

El resultado del modelo exhaustivo de características diseñado para evaluar los archivos con presencia en Internet puede observarse en la clasificación siguiente, donde aparecen, en primer lugar, los indicadores de características generales $\mathrm{y}$, en segundo lugar, las características específicas agrupadas en sus criterios respectivos y ordenadas según el proceso archivístico. Se manifiesta que todas las características tienen carácter positivo.

Clasificación de características para evaluar los sitios web de archivos.

\section{CARACTERÍSTICAS GENERALES}

1. Los objetivos del sitio web se expresan con claridad

2. Tiene una URL correcta, clara y fácil de recordar

3. Posee una URL estable

4. La estructura general del sitio web está orientada al usuario

5. Aporta un diseño general del sitio web coherente

6. Se permite la visualización del sitio con navegadores estándar

7. Tiene un acceso rápido y regular

8. Aparece la fecha de creación intelectual del recurso

9. El logotipo es significativo, identificable y suficientemente visible

10. Ofrece algún enlace con información sobre el sitio web, 'webmaster'

11. Está dirigido a un ámbito geográfico o lingüístico concreto

12. Proporciona alguna información nueva o innovadora

13. Se cuida la gramática y la ortografía

14. Se menciona el área geográfica que abarca

15. Posee un lenguaje claro y conciso

16. Es amigable, familiar y cercano 


\begin{tabular}{|l|}
\hline 17. 1 párrafo $=1$ idea \\
\hline 18. Dispone de un mapa sensible \\
\hline 19. Contiene rótulos significativos \\
\hline 20. Funcionan los enlaces de la home page, sean internos o externos \\
\hline 21. Tiene enlaces fácilmente reconocibles como tales \\
\hline 22. No tiene sobrecarga informativa \\
\hline
\end{tabular}

\begin{tabular}{|c|}
\hline CARACTERÍSTICAS ESPECÍFICAS \\
\hline Criterio: IDENTIFICACIÓN \\
\hline $\begin{array}{c}\text { 23. Indica los procedimientos que se siguen a la hora de identificar } \\
\text { documentos }\end{array}$ \\
\hline
\end{tabular}

\section{Criterio: VALORACIÓN}

24. Contiene ficha de valoración y descripción de organismos

25. Da acceso al inventario documental

26. Permite descarga del inventario documental

\section{Criterio: ELIMINACIÓN DOCUMENTAL}

27. Contiene tablas de valoración documental

28. Contiene calendario de conservación

29. Existe creada una Comisión Calificadora de Documentos

30. Tiene inventario de eliminación

31. Contiene acta de eliminación

32. Tiene memoria de eliminación

33. Dispone de solicitud de eliminación

34. Contiene autorización de la eliminación

35. Muestra diagramas sobre el proceso de expurgo

\section{Criterio: DESCRIPCIÓN}

36. Permite la visualización del cuadro de clasificación a través de la web

37. Sigue la Norma ISAAR (CPF) (International Standard Archival Authority Record for Corporate Bodies, Persons, and Families)

38. Sigue la Norma ISDIAH (International Standard for Describing Institutions with Archival Holdings)

39. Sigue la Norma ISAD(G) (General International Standard Archival Description)

40. Sigue la Norma ISO (International Organization for Standardization) 15489. Información y documentación. Gestión de documentos

41. Sigue la Norma NEDA (Norma Española de Descripción Archivística) 


\section{Criterio: INSTRUMENTOS DE DESCRIPCIÓN}

42. Dispone de guía

43. Dispone de inventario

44. Dispone de catálogo

45. Contiene otros instrumentos auxiliares

\section{Criterio: ORGANIZACIÓN}

\section{Sub-criterio: Clasificación}

46. Dispone de cuadro de clasificación de fondos

47. Indica el tipo de clasificación que sigue el archivo

\section{Sub-criterio: Ordenación}

48. Indica el tipo de ordenación que sigue el archivo

\section{Criterio: INSTALACIÓN}

49. Muestra las unidades de instalación que utiliza

50. Informa sobre los metros lineales de documentación o volumen de la colección

\section{Criterio: ACCESO A LA INFORMACIÓN}

\section{Sub-criterio: Préstamo}

51. Ofrece el servicio de préstamo de documentos a usuarios internos

52. Ofrece el servicio de préstamo de documentos a usuarios externos

53. Aparece la normativa del servicio de préstamo

54. Da acceso a la normativa de préstamo

55. Permite la descarga de modelos normalizados para usuarios internos mediante clave de acceso

56. Permite la descarga de modelos normalizados para usuarios externos al archivo

57. Contiene modelo de autorización para gestores de la documentación

58. Contiene modelo de solicitud de préstamo

59. Se aporta el modelo de documento de control o testigo de préstamo

60. Ofrece diagramas sobre el proceso de préstamo

61. Indica las instrucciones para cumplimentar modelos normalizados

\section{Sub-criterio: Consulta}

62. Se ofrece servicio de consulta de documentos

63. Se ofrece modelo de consulta de documentos 


\section{Criterio: TRANSFERENCIA DE INFORMACIÓN}

64. Contiene instrucciones sobre cómo realizar una transferencia

65. Tiene modelo de relación de entrega

66. Existe modelo de diligencia de cotejo

67. Permite la descarga de modelos normalizados para usuarios internos mediante clave de acceso

68. Permite la descarga de modelos normalizados para usuarios externos al archivo

69. Recoge un calendario de transferencias o, en su defecto, una previsión de futuras transferencias al archivo

\section{Criterio: NUEVAS TECNOLOGÍAS EN LOS ARCHIVOS}

70. Utiliza algún software para la gestión de archivos como

71. Utiliza software libre

72. Posee una base de datos que recoge los fondos del archivo

73. Permite el acceso a la base de datos a usuarios internos

74. Permite el acceso a la base de datos a usuarios externos

75. Contiene sus fondos digitalizados

76. Se permite la descarga del fondo digitalizado a usuarios internos

77. Se permite la descarga del fondo digitalizado a usuarios externos

\section{Criterio: CERTIFICACIONES}

78. Contiene certificaciones de calidad por alguna entidad certificadora

79. Contiene certificaciones por otras entidades

80. Posee indicadores de calidad

\section{Criterio: PERSONAL DE LA INSTITUCIÓN}

81. Se pueden visualizar datos de contacto

\section{Criterio: LEGISLACIÓN}

82. Hace referencia a la legislación nacional vigente

83. Hace referencia a la legislación autonómica vigente

84. Hace referencia a la legislación local vigente

\section{Criterio: INFORMACIÓN CULTURAL}

85. Contiene ponencias

86. Contiene artículos

87. Realiza exposiciones 
88. Contiene bibliografía

89. Contiene "Pieza del mes"

90. Contiene novedades sobre archivos

\section{Criterio: ENLACES}

91. Posee enlaces a asociaciones profesionales

92. Contiene enlaces a manuales

93. Posee otros enlaces de interés

\section{Criterio: OTRA INFORMACIÓN}

94. Posee contador de usuarios o visitas

95. Aparecen encuestas de satisfacción

96. Contiene el manual de normas y procedimientos del archivo

97. Aparece el organigrama de la institución a la que pertenece el archivo

98. El archivo está incluido en el organigrama de la entidad

99. Contiene glosario de términos

100. Contiene carta de servicios

A continuación, se explica de forma detallada cada una de las características del modelo diseñado:

\subsection{CARACTERÍSTICAS GENERALES}

1. Los objetivos del sitio web se expresan con claridad: se deben indicar en la página de inicio que cumple la función de 'escaparate' del sitio web.

2. Tiene una URL correcta, clara y fácil de recordar: por ejemplo, $\mathrm{http}: / / \mathrm{www}$.archivohistoricodepotes.es/.

3. Posee una URL estable: es un factor importante a tener en cuenta ya que ayuda en la localización del recurso.

4. La estructura general del sitio web está orientada al usuario: no se debe calcar la estructura interna de la organización, ya que al usuario no le interesa cómo funciona o se organiza físicamente la misma.

5. Aporta un diseño general del sitio web coherente: se debe mantener una coherencia y uniformidad en las estructuras y colores de todas las páginas para que el usuario no se desoriente en su navegación.

6. Se permite la visualización del sitio con navegadores estándar: tipo Internet Explorer, Mozilla Firefox, Google Chrome, etc.

7. Tiene un acceso rápido y regular. 
8. Aparece la fecha de creación intelectual del recurso: las fechas que se muestran en la web deben corresponder a noticias, actualizaciones, eventos, etc. no a la fecha del sistema del usuario.

9. El logotipo es significativo, identificable y suficientemente visible: un logotipo es la marca distintiva de una compañía, un producto, un servicio o una gama de productos o servicios de una misma fuente. Se entiende por "logotipo" al que usa solo tipografía como marca, "isotipo" al que usa solo un icono como marca e "isologo" al que usa tipografía e icono.

10. Ofrece algún enlace con información sobre el sitio web, 'webmaster': debe existir una persona encargada de la implementación, configuración, diseño, mantenimiento, promoción y la mejora continuada del sitio web.

11. Está dirigido a un ámbito geográfico o lingüístico concreto: hace referencia a la lengua que aparece en la web.

12. Proporciona alguna información nueva o innovadora: ofrece información primaria.

13. Se cuida la gramática y la ortografia: respeta las normas de puntuación, ortografía, concordancia de los verbos, etc.

14. Menciona el área geográfica que abarca: hace referencia a la localidad en la que se encuentra emplazado el archivo.

15. Posee un lenguaje claro y conciso: se debe evitar usar un lenguaje corporativista. Así mismo, hay que prestarle especial atención al idioma, y ofrecer versiones del sitio en diferentes idiomas cuando sea necesario.

16. Es amigable, familiar y cercano: no se debe utilizar un lenguaje constantemente imperativo o mensajes crípticos.

17. 1 párrafo $=1$ idea: cada párrafo es un objeto informativo, trasmite ideas, mensajes, etc. Se deben evitar párrafos vacíos o varios mensajes en un mismo párrafo.

18. Dispone de un mapa sensible: contiene un mapa web o sitemap que nos permite navegar con más facilidad por la web, además de ofrecernos una estructura jerárquica del sitio web.

19. Contiene rótulos significativos: un rótulo significativo es aquel que da a conocer con propiedad una cosa. Por eso, es importante evitar rótulos del tipo "haga clic aqui".

20. Funcionan los enlaces de la "home page", sean internos o externos: los enlaces internos enlazan las páginas que componen un mismo sitio web; los enlaces externos permiten enlazar páginas de sitios web diferentes.

21. Tiene enlaces fácilmente reconocibles como tales: los enlaces deben ser reconocidos como una unidad.

22. No tiene sobrecarga informativa: se consigue haciendo un uso correcto de colores, efectos tipográficos y agrupaciones para discriminar información. Al igual que en los elementos de un menú de navegación, los 
grupos diferentes de objetos informativos de una página no deberán superar el número $7 \pm 2$.

\subsection{CARACTERÍSTICAS ESPECÍFICAS DE UN SITIO WEB DE ARCHIVOS}

\section{Identificación}

23. Indica los procedimientos que se siguen a la hora de identificar documentos: En una web dedicada a archivos es necesario indicar la sistematización de las categorías administrativas y archivísticas en que se sustenta la estructura de un fondo (Diccionario de Terminología Archivística, 1995).

\section{Valoración}

Para una correcta formalización de este indicador se deben justificar los distintos valores (Schellenberg, 1958), incluyendo la posible inexistencia de alguno de ellos. Igualmente hay que indicar los plazos de tiempo en los que permanece cada valor (Cruz Mundet, 2011).

24. Contiene ficha de valoración y descripción de organismos: es la fase del tratamiento archivístico que consiste en analizar y determinar los valores primarios y secundarios de las series documentales, fijando los plazos de transferencia, acceso y conservación o eliminación total o parcial (Diccionario de Terminología Archivística, 1995).

25. Da acceso al inventario documental: El inventario es el instrumento que describe las unidades de instalación y/o las unidades documentales de una serie o series, respetando su estructura. Las entradas descriptivas de los inventarios, están formadas por: signatura, descripción, fechas extremas y código de clasificación.

26. Permite descarga del inventario documental: el inventario puede ser descargado por el usuario desde el sitio web en formato word o pdf.

\section{Eliminación documental}

La eliminación o expurgo, es el procedimiento archivístico que consiste en la identificación de los documentos que se van a destruir conforme a los plazos establecidos en la fase de valoración (Diccionario de Terminología Archivística, 1995).

27. Contiene tablas de valoración documental: las tablas de valoración son un instrumento que establece los plazos de conservación y la transferencia de una serie determinada junto a los requisitos para el acceso (Heredia Herrera, 2011). 
28. Contiene calendario de conservación: es el instrumento oficial de referencia a la hora de una propuesta de eliminación o de justificar una eliminación directa, y para cumplir los plazos de transferencia (Heredia Herrera, 2011). Hay diversos modelos para los calendarios de conservación, pero todos coinciden en que deben contener, al menos, lo siguiente: 1) denominación de la serie (y su código), 2) nombre del organismo productor, 3) Conservación (sí/no) y plazos de eliminación, 4) Muestreo (tipo), 5) Observaciones (Cruz Mundet, 2011).

29. Informa sobre la Comisión Calificadora de Documentos: es el órgano que tiene como competencias específicas el estudio y dictamen sobre los plazos de transferencia, posible eliminación, acceso y utilización de los documentos y series documentales previamente valorados, y cualquier asunto sobre materia archivística relacionada con sus competencias.

30. Tiene inventario de eliminación: de acuerdo con los calendarios de conservación, el gestor jefe del Archivo/Unidad Administrativa establece la relación de documentación a eliminar en la memoria de eliminación.

31. Contiene acta de eliminación: la importancia del acta de eliminación reside en la constancia de acto de destrucción autorizada de documentos.

32. Tiene memoria de eliminación: la memoria de eliminación es el documento en el que se refleja un listado de todas las actas de eliminación que se han realizado.

33. Dispone de solicitud de eliminación: el responsable de la unidad administrativa solicita al archivo autorización para destruir la documentación que indica en el formulario de solicitud de eliminación.

34. Contiene autorización de la eliminación: esta etapa sólo se da si la documentación a eliminar se encuentra en un archivo de gestión.

35. Muestra diagramas sobre el proceso de expurgo: en esta característica se valora que existan diagramas sobre expurgo o eliminación de documentación que faciliten la comprensión del procedimiento.

\section{Descripción}

La descripción documental comprende no solo el análisis de los documentos (...), sino también los datos para su localización. La descripción es necesaria tanto en un archivo administrativo como en un archivo histórico, aunque su práctica pueda presentar algunas variantes (Heredia Herrera, 1989).

Para la descripción de las siguientes Normas se ha seguido el "Directorio español de recursos electrónicos que difunde normas técnicas archivisticas y documentos profesionales" del Ministerio de Cultura (http://www.mcu.es/archivos/CE/RecProf/NormasDocumentos.html). 
36. Permite la visualización del cuadro de clasificación a través de la web: la importancia de esta característica radica en que el usuario que navega por la web de un archivo puede formarse una idea de los fondos que alberga dicho archivo.

37. Sigue la Norma ISAAR (CPF) (International Standard Archival Authority Record for Corporate Bodies, Persons, and Families, Norma Internacional sobre los Registros de Autoridad de Archivos relativos a Instituciones, Personas y Familias). Los registros de autoridad de archivos se pueden utilizar para: describir una institución, persona o familia como unidades dentro de un sistema de descripción archivístico; controlar la creación y utilización de los puntos de acceso en las descripciones archivísticas; documentar las relaciones entre diferentes productores de documentos y entre estas entidades y los documentos creados por ellas, y/o otros recursos que les conciernen.

38. Sigue la Norma ISDIAH (International Standard for Describing Institutions with Archival Holdings, Norma Internacional para Describir Instituciones que Custodian Fondos de Archivo): esta norma establece las reglas generales para la normalización de la descripción de instituciones que conservan fondos de archivo, proporcionando directrices prácticas para identificar y contactar con las instituciones que tienen fondos de archivo, acceder a los mismos y acceder a los servicios que la institución ofrece.

39. Sigue la Norma ISAD $(G)$ (General International Standard Archival Description, Norma Internacional General de Descripción Archivística): esta norma constituye una guía general para la elaboración de descripciones archivísticas compatibles a nivel nacional e internacional, por ello, en el texto de la norma se especifica que: "Debe utilizarse juntamente con las normas nacionales existentes o como base para el desarrollo de las normas nacionales". Esta norma es, ante todo, un punto de partida.

40. Sigue la Norma ISO (International Organization for Standardization) 15489. Información y documentación. Gestión de documentos: esta norma regula la gestión de documentos de las organizaciones que los han producido, ya sean públicas o privadas, para clientes externos e internos. Así mismo, también regula la gestión de los documentos con los sistemas electrónicos para la conservación de archivos en diferentes.

41. Sigue la Norma NEDA (Norma Española de Descripción Archivística): esta norma constituye una herramienta normativa técnica, aplicable a cualquier tipo de archivo y a cualquier tipo de recurso archivístico (textual, gráfico, audiovisual y electrónico).

\section{Instrumentos de descripción}

42. Dispone de guia: instrumento de referencia que describe globalmente fondos documentales indicando las características fundamentales de los 
mismos: organismos que los originan, series que los forman, fechas extremas que comprenden y volumen de la documentación. Además, recoge la información sobre el archivo, su historia y formación, horarios y servicios a los usuarios (Diccionario de Terminología Archivística, 1995).

43. Dispone de inventario: instrumento de referencia que describe las series documentales de un fondo, siguiendo su organización y que, por motivos de localización, se encuentran fraccionadas en unidades de instalación. El inventario debe recoger, imprescindiblemente, una introducción histórica explicativa de la evolución del organismo productor de los documentos y el cuadro de clasificación del fondo descrito. Los asientos del inventario recogen la signatura de la unidad de instalación, el nombre de la serie y las fechas que comprende. Debe completarse con los índices correspondientes (Diccionario de Terminología Archivística, 1995).

44. Dispone de catálogo: instrumento de referencia en el que, con la finalidad de informar sobre una materia u objeto específico, se describe unidades documentales relacionadas por su autor en razón de una afinidad temática, cronológica, paleográfica o formal o por otro criterio subjetivo establecido de antemano (Diccionario de Terminología Archivística, 1995).

45. Contiene otros instrumentos auxiliares: registro de entrada y salida de documentos, relaciones de entrega, registro de transferencias, registro de préstamos, registro topográfico, registro gráfico de depósitos, registro de usuarios e investigadores.

\section{Organización}

Mediante las etapas de clasificación y ordenación, este proceso aplica las conclusiones establecidas en la fase de identificación a la estructura de un fondo (Diccionario de Terminología Archivística, 1995).

\section{Clasificación}

46. Dispone de cuadro de clasificación de fondos: el cuadro de clasificación refleja la organización de un fondo documental o de la totalidad de los fondos de un archivo y aporta los datos esenciales de su estructura (denominación de secciones y series, fechas extremas, etc.). Plasma a la perfección los fondos que contiene un archivo. Es preciso distinguir siempre dos niveles o estadios: el primero se identifica con la estructura o funcionamiento de la institución (por ejemplo su organigrama: órganos y funciones) y corresponde a las secciones y subsecciones o bien grupos o subgrupos; el segundo nivel equivale a las series documentales (Heredia Herrera, 1989).

47. Indica el tipo de clasificación que sigue el archivo: se deben establecer las categorías y grupos que reflejan la estructura jerárquica del fondo. Es el primer paso del proceso de organización, dentro de la fase del tratamiento 
archivístico denominada identificación. Existen dos tipos de clasificaciones: orgánica y funcional (Delgado Gómez y Cruz Mundet, 2011) y Heredia Herrera $(1989,2011)$.

\section{Ordenación}

48. Indica el tipo de ordenación que sigue el archivo: la ordenación documental es la ubicación física de los documentos dentro de la respectiva clasificación y orden previamente definido y acordado. Dependiendo del fondo que posea el archivo, este debe estar ordenado de forma alfabética, numérica o cronológica (Heredia Herrera, 2011) o alfabética, cronológica, geográfica, decimal o por materias (Lodolini, 1993).

\section{Instalación}

Es la operación que consiste en la colocación ordenada de las unidades de instalación en las estanterías del depósito del archivo tras el ingreso de los documentos (Heredia Herrera, 2011).

49. Aparece identificado el volumen en unidades de instalación: esta característica muestra las unidades de instalación (cajas) cuantificables de las que consta el fondo.

50. Informa sobre los metros lineales de documentación o volumen del fondo: junto con la anterior, es una de las características que muestra el volumen de documentación que custodia el archivo.

Es conveniente disponer de indicadores de crecimiento anual de las series documentales que se evalúan. Se debería hacer constar los metros lineales de crecimiento al año, de forma aproximada, teniendo en cuenta los datos proporcionados por los propios productores o por las transferencias de los años precedentes al archivo (Cruz Mundet, 2011).

\section{Acceso a la información}

Es conveniente que se indiquen los criterios de acceso y consulta de la documentación, así como las posibles restricciones al acceso. Los motivos de restricción suelen estar tipificados en las normativas legales de los estados (Cruz Mundet, 2011). Hay que tener presente la siguiente legislación:

- Constitución de 1978 que recoge en su artículo 105.b "el acceso de los ciudadanos a los archivos y registros administrativos..."

- Ley de Patrimonio Histórico Español (Ley 16/1985, de 25 de junio)

- Ley del Régimen Jurídico de las Administraciones Públicas y del Procedimiento Administrativo Común (Ley 30/1992 de 26 de noviembre).

- Ley Orgánica 15/1999, de 13 de diciembre, de Protección de Datos. 


\section{Préstamo}

51. Ofrece el servicio de préstamo de documentos a usuarios internos: el hecho de que el archivo ofrezca el préstamo desde su web ayuda al conocimiento de los fondos que custodia.

52. Ofrece el servicio de préstamo de documentos a usuarios externos: este servicio resulta apropiado para que el archivo ofrezca libremente (aunque siempre sujeto a restricciones) el acceso a sus fondos.

53. Aparece la normativa del servicio de préstamo: deben aparecer en la web una serie de normas que regulen el préstamo de documentos (por ejemplo, las peticiones de consulta o de préstamo de los documentos solicitados se formalizarán mediante impreso normalizado; los préstamos siempre se efectuarán a partir de expedientes enteros, nunca se servirán partes de expedientes o documentos sueltos de una unidad documental; el personal del Archivo Universitario es el único autorizado para realizar reproducciones de documentos, etc.).

54. Da acceso a la normativa de préstamo: normalmente esta normativa suele aparecer implícita en el reglamento del archivo.

55. Permite la descarga de modelos normalizados para usuarios internos mediante clave de acceso: se puede considerar una característica secundaria, ya que lo realmente importante es que exista el servicio de préstamo en la web.

56. Permite la descarga de modelos normalizados para usuarios externos al archivo: se puede considerar una característica secundaria, ya que lo realmente importante es que exista el servicio de préstamo en la web.

57. Contiene modelo de autorización para gestores de la documentación: contiene modelo de solicitud de préstamo; se aporta el modelo de documento de control o testigo de préstamo (nombre del solicitante, datos de la documentación y datos de control interno del préstamo).

58. Contiene modelos de solicitud de préstamo: este documento se trata de un modelo en el cual queda reflejada la petición de documentación por parte del usuario al archivo.

59. Se aporta el modelo de documento de control o testigo de préstamo: de este modelo se rellenarán tres ejemplares, y será el documento que permita tener localizada la documentación (un ejemplar irá grapado al expediente que se solicite en préstamo, el segundo ejemplar se introducirá en una carpeta donde estarán recogidas las salidas de documentación del archivo, y el tercer ejemplar se introducirá en la caja de la cual se extrajo el expediente).

60. Ofrece diagramas sobre el proceso de préstamo: los diagramas de flujo favorecen la comprensión del proceso al mostrarlo como un dibujo (un 
buen ejemplo de ello es el diagrama usado para el proceso de préstamo en el Archivo de la Universidad de Zaragoza ${ }^{1}$ ).

61. Indica las instrucciones para cumplimentar modelos normalizados: para facilitar la comprensión del usuario, es preciso que los modelos normalizados estén acompañados de las correspondientes instrucciones para cumplimentarlos.

\section{Consulta}

62. Se ofrece servicio de consulta de documentos: la consulta es el examen directo de la documentación por parte de los usuarios (investigadores, Administración, etc.), sin que ello implique necesariamente la autorización para que éstos reproduzcan y/o divulguen total o parcialmente los datos contenidos en los documentos (Diccionario de Terminología Archivística, 1995)..

63. Se ofrece modelo de consulta de documentos: al igual que en el caso del préstamo, lo realmente importante es que exista el servicio de consulta en la web del archivo.

\section{Transferencia de información}

Procedimiento habitual de ingreso de fondos en un archivo mediante traslado de las fracciones de series documentales, una vez que éstas han cumplido el plazo de permanencia fijado por las normas establecidas en la valoración para cada una de las etapas del ciclo vital de los documentos (Diccionario de Terminología Archivística, 1995).

64. Permite realizar transferencias online y contiene instrucciones sobre cómo realizar una transferencia: la web del archivo ofrece la posibilidad de gestionar las transferencias y además proporciona las instrucciones sobre cómo realizar una transferencia a dicho archivo.

65. Tiene modelo de relación de entrega (también denominada hoja o relación de transferencia): es importante que la web del archivo ofrezca el modelo de relación de entrega que se utiliza en el Archivo Receptor, de tal forma que al Archivo Remitente le resulte más cómodo realizar la transferencia, sin la necesidad de haber mantenido el contacto previo con el Archivo Receptor.

66. Existe modelo de diligencia de cotejo: el cotejo de documentos es la operación integrada en el proceso de ingreso de documentos consistente en

1 Diagrama para el Procedimiento de Préstamo de Documentos del Archivo de la Universidad de Zaragoza (http://wzar.unizar.es/uz/arch/). 
comprobar la adecuación de los documentos recibidos, en número y en contenido, con la propuesta de ingreso (Heredia Herrera, 2011). Se trata de un valor añadido que puede tener la web del archivo.

67. Permite la descarga de modelos normalizados para usuarios internos mediante clave de acceso: la descarga de los modelos normalizados permitirá al usuario conocer de primera mano el modelo de relación de entrega que utiliza el Archivo Receptor.

68. Permite la descarga de modelos normalizados para usuarios externos al archivo: la descarga de los modelos normalizados permitirá al usuario familiarizarse con los modelos que se utilizan en el Archivo Receptor.

69. Recoge un calendario de transferencias o en su defecto una previsión de futuras transferencias al archivo: es un instrumento de uso interno dentro del archivo que le permite planificar y establecer los plazos de las transferencias. Algunos archivos cuenta también con un modelo de transferencias previstas, donde se recogen los ingresos que se realizarán en el archivo a lo largo del año.

\section{Nuevas tecnologías en los archivos}

70. Utiliza algún software para la gestión de archivos: como Archivo 3000, Albalá, AlbalaNET, Anser, Archon, ARGES, CLARA, DIGIARCH, EDAS Archivo Físico, FLORA Archiv, ICA-AtoM, IRGES, Pinakes Archives etc. (Criteria for software evaluation: a checklist for archivists, 1993).

71. Utiliza software libre: existen algunos archivos en los que se utilizan programas en software libre para su gestión (Sistema Operativo GNU/Linux) que ofrecen la posibilidad de ejecutar, copiar, distribuir, estudiar, cambiar y mejorar el software.

72. Posee una base de datos que recoge los fondos del archivo: es muy positivo que exista una base de datos que recoja los fondos pormenorizados del archivo y se exponga a través de la web (Perpinyá Morera, 2000).

73. Permite el acceso a la base de datos a usuarios internos: es necesario que el usuario pueda observar los fondos de los que dispone el archivo a través de una base de datos pormenorizada que ofrezca la web del archivo. De esta manera, se evitará el desplazamiento del usuario a la parte física del archivo donde se encuentren los fondos.

74. Permite el acceso a la base de datos a usuarios externos: es necesario que el usuario pueda observar los fondos de los que dispone el archivo a través de una base de datos pormenorizada que ofrezca la web del archivo. De esta manera, se evitará el desplazamiento del usuario a la sede física del archivo.

75. Contiene sus fondos digitalizados: un archivo que contenga sus fondos digitalizados permite la difusión del mismo y conserva su documentación. 
76. Se permite la descarga del fondo digitalizado a usuarios internos: se permite a los usuarios internos acceder y descargar los fondos digitalizados de su interés.

77. Se permite la descarga del fondo digitalizado a usuarios externos: a través de esta característica se permite a cualquier usuario acceder a los fondos concretos sobre los que desea investigar, favoreciendo su descarga.

\section{Certificaciones}

Las certificaciones de calidad son emitidas por entidades certificadoras. La Entidad Nacional de Acreditación (ENAC) es la encargada en España de reconocer y determinar si una organización es o no competente para certificar a otras.

78. Contiene certificaciones de calidad por alguna entidad certificadora: el archivo contiene certificados de calidad emitidos por entidades certificadoras como Asociación Española de Normalización (AENOR), Asociación Española para la Calidad (AEC), iQUA (Agencia de Calidad de Internet), Deloitte, etc.

79. Contiene certificaciones por otras entidades: también es preciso valorar las web de archivos que hayan sido distinguidas con menciones de calidad de entidades no reconocidas como certificadoras.

80. Posee indicadores de calidad: es muy positivo que los sitios web de archivos posean otros indicadores de calidad además de las certificaciones (por ejemplo, premios, citas recibidas, etc.) (Heredia Herrera, 2007).

\section{Personal de la institución}

81. Se pueden visualizar datos de contacto: es necesario que se proporcione algún mecanismo para ponerse en contacto con la institución (email, teléfono, dirección postal, fax...).

\section{Legislación}

82. Hace referencia a la legislación nacional vigente: sería suficiente con que se reflejara la legislación nacional que afecta al archivo.

83. Hace referencia a la legislación autonómica vigente: debería reflejar la legislación autonómica o regional que afecte al archivo.

84. Hace referencia a la legislación local vigente: debería reflejar la legislación local que afecte al archivo.

\section{Información cultural}

Las características que vienen a continuación han sido consideradas como un proceso evolutivo en la concepción funcional de los archivos en el ámbito sociocultural. Cabe destacar la importancia que tiene el hecho de que un archivo contenga información sociocultural en cualquiera de sus ámbitos. Las actividades 
de difusión que pueden llevar a cabo los archivos son muchas y variadas, desde las clásicas exposiciones, cursos, jornadas, congresos y conferencias, hasta las vertientes pedagógicas más innovadoras (talleres, dramatizaciones...) con más incidencia en el mundo educativo.

85. Contiene ponencias

86. Contiene artículos

87. Realiza exposiciones

88. Contiene bibliografía

89. Contiene "Pieza del mes"

90. Contiene novedades sobre archivos

\section{Enlaces}

91. Posee enlaces a asociaciones profesionales: el archivo debe contener un amplio abanico de enlaces de varias temáticas y, además, enlaces hacia asociaciones profesionales especializadas en archivos.

92. Contiene enlaces a manuales: el archivo debe contener enlaces hacia manuales de referencia en la disciplina.

93. Posee otros enlaces de interés: es importante que el archivo contenga accesos a directorios de archivos europeos, a otros archivos, al Consejo Internacional de Archivos, etc.

\section{Otra información}

94. Posee contador de usuarios o visitas: este instrumento permite conocer el nivel de acceso o visitas que tiene la web a lo largo de un determinado período de tiempo. Así, se puede medir la importancia que adquieren los fondos del archivo para los usuarios que acceden a él.

95. Aparecen encuestas de satisfacción: se trata de una información añadida para una web de archivos.

96. Contiene el manual de normas y procedimientos del archivo: esta característica puede ser prescindible en el caso de que aparezcan definidos en el sitio web los procesos que se siguen, tales como préstamo, consulta, transferencia, etc.

97. Aparece el organigrama de la institución a la que pertenece el archivo: el organigrama se define como la representación gráfica de la estructura orgánica de una institución o de una de sus áreas y debe reflejar en forma esquemática la descripción de las unidades que la integran, su respectiva relación, niveles jerárquicos y canales formales de comunicación.

98. El archivo está incluido en el organigrama de la entidad: es importante que el organismo al que pertenece el archivo lo considere y reconozca como parte integrante del mismo. 
99. Contiene glosario de términos: es importante que aparezca en la web una lista de términos que permitan al usuario familiarizarse con el entorno.

100. Contiene carta de servicios: las cartas de servicios son documentos que tienen por objeto informar a la ciudadanía acerca de las cualidades de los servicios públicos. De modo general suelen expresar: la naturaleza, contenido, características y formas de prestación de los servicios, la determinación de los niveles o estándares de calidad en la provisión de los servicios, los mecanismos de consulta a la ciudadanía acerca de los servicios que aquella demande y de sus sugerencias y opiniones para la mejora de los mismos, y el sistema de evaluación de la calidad (Grupo de trabajo sobre calidad en los Archivos Universitarios, 2012).

\section{CONCLUSIONES}

De la investigación realizada se obtienen las siguientes conclusiones:

1. Aunque existen bastantes trabajos sobre indicadores web(o)métricos de análisis de características en el contexto de las unidades informativas, la mayoría de ellos se han centrado en el ámbito de las bibliotecas. En el caso de los sitios web de archivos, sin embargo, existen pocas investigaciones sobre análisis de sus características y, además, los modelos desarrollados en ellas utilizan, en general, indicadores demasiado universales que se pueden aplicar en ámbitos muy heterogéneos y no específicos de archivos.

2. Es posible desarrollar un modelo exhaustivo para la evaluación de los sitios web de archivos centrado en el análisis de las características específicas de los mismos. Así, en el presente trabajo se diseña un modelo de 100 características donde el $78 \%$ de las mismas son de carácter específico para evaluar la calidad de los archivos con presencia en Internet. Este hecho corrobora la hipótesis de partida de la investigación.

3. Internet ha conllevado el inexorable avance hacia el archivo virtual y el acceso remoto a la información, lo que ha provocado un cambio en el perfil de los usuarios, diversificándose en cuanto a la tipología de los mismos. Del tradicional, y prácticamente exclusivo, investigador profesional se ha pasado a la eclosión de usuarios, tanto en número como en tipo (usuarios internos, externos, estudiantes, ciudadanos sin formación científica específica, etc.). A ello ha contribuido el fomento de la participación ciudadana, la transparencia administrativa, así como la facilidad de acceso a la información. Este último aspecto se ha querido reflejar en el modelo 
diseñado, donde el $16,7 \%$ del total de de las características específicas se agrupan bajo el criterio "Acceso a la información".

\section{BIBLIOGRAFÍA}

AL-DWAIRI, K.; FABA PÉREZ, C.; VARGAS QUESADA, B. “Indicadores Webmétricos de carácter Formal para Evaluar el Posicionamiento de las Universidades: el caso de los Países Árabes”. Investigación Bibliotecológica: archivonomía, bibliotecología e información, 2010, 24/52:145-171.

CABALLERO CORTÉS, L; FABA PÉREZ, C.; MOYA ANEGÓN, F. "Evaluación comparativa de la accesibilidad de los espacios web de las bibliotecas universitarias Españolas y Norteamericanas". Investigación Bibliotecológica: archivonomía, bibliotecología e información, 2009, 47/23:45-66.

CHAÍN NAVARRO, C; GARCÍA GONZÁLEZ, M. "Los contenidos de las web de los archivos históricos estatales. El ciudadano más cerca de la información". Cuadernos de Documentación Multimedia, 2009, 20:5-24.

CHAIIN NAVARRO, C.; MÁS BLEDA, A. "Los archivos nacionales hispanoamericanos y la gestión de información de sus sedes web". Biblios: Revista de Bibliotecología y Ciencias de la Información, 2009, 35:1-23.

CHAO, H. "Assessing de quality of academic libraries on the Web: the development and testing of criteria". Library \& Information Science Research, 2002, 24:169-194.

CLAUSEN, H. "Evaluation of library Web sites: the Danish case". The Electronic Library, 1999, 17/2:83-87.

CLYDE, L. A. "School library Web sites: 1996-2002". The Electronic Library, 2004, 22/2:158-167.

CONDE VILLAVERDE, M. L. Manual de tratamiento de archivos administrativos. Madrid : Dirección de Archivos Estatales, 1992.

CRAVEN, J. "Electronic Access for All: Awareness in Creating Accessible Web Sites for the University Library". Disability and Information Systems in Higher Education (DISinHE) [en línea], 2000. Disponible en

"http://www.dmag.org.uk/resources/casestudies/cravenfull.asp" [Consulta: 10 mayo 2011].

CRESPO, S. "Archivos Histórico Provinciales en la red: análisis y evaluación”. Boletín de la ANABAD, 2007,1/2:497-512.

CRITERIA FOR SOFTWARE EVALUATION: A CHECKLIST FOR ARCHIVISTS. London: Society of Archivists, 1993.

CRUZ MUNDET, J. R. Manual de Archivística. Madrid: Fundación Germán Sánchez Ruipérez, 1994. 
CRUZ MUNDET, J. R. (Dir.). Administración de documentos y archivos: Textos Fundamentales. Madrid: Coordinadora de Asociaciones de Archiveros y Gestores de Documentos (CAA), 2011.

CUADRADO FERNÁNDEZ, O.; GÓMEZ DOMÍNGUEZ, D. "Los archivos municipales españoles en línea: evaluación del contenido de sus webs". Actas del IV Jornadas de Archivos Electrónicos, Priego de Córdoba, 2002. [en línea]. Disponible en "http://www.ugr.es/ vjarchiv/images/David.doc" [Consulta: 14 Junio 2011].

DELGADO GÓMEZ, P.; CRUZ MUNDET, J. R.. Diccionario de Archivística: (con equivalencias en inglés, francés, alemán, portugués, catalán, euskera y gallego). Madrid: Alianza, 2011.

DICCIONARIO DE TERMINOLOGÍA ARCHIVÍSTICA. Ministerio de Cultura. Subdirección General de los Archivos Estatales, 1995.

DUPLÁ DEL MORAL, A. Manual de archivos de oficina para gestores: Comunidad de Madrid. Madrid: Marcial Pons, 1997.

FABA PÉREZ, C.; GUERRERO BOTE, V.; MOYA ANEGÓN, F. Fundamentos y técnicas cibermétricas. Mérida: Junta de Extremadura, 2004.

FALK, H. "Library web site innovations". The Electronic Library, 1999, 17/5:323-328.

FERNÁNDEZ GIL, P. Manual de Organización de Archivos de Gestión en las Oficinas Municipales. Granada: CEMCI, 1997.

GIL GARCÍA, P. "Tejiendo Archivos: lo que la WWW puede hacer por un archivo". Biblos, 2001, 3/10.

GIL GARCÍA, P. "Panorama de los archivos universitarios en la Red: el mapa web de archivos de universidades españolas". Archivos Universitarios e Historia de las Universidades, 2003, 153-166.

GRUPO DE TRABAJO SOBRE CALIDAD EN LOS ARCHIVOS UNIVERSITARIOS. Estructura y contenidos mínimos de cartas de servicios, de acuerdo a la NORMA UNE 93200:2008. En: XVIII Jornadas de la Conferencia de Archiveros de Universidades, 2012 [en línea]. Disponible en:

"http://cau.crue.org/export/sites/Cau/Quehacemos/gruposdetrabajo/calidad/modelo carta_servicios_2010.pdf' [Consulta: 30 Julio 2012].

HASAN MONTERO, Y.; MARTÍN FERNÁNDEZ, F. J. "Guía de Evaluación Heurística de Sitios Web". No Solo Usabilidad. [en línea], 2003, 2. Disponible en "http://www.nosolousabilidad.com/articulos/heuristica.htm" [Consulta: 12 Abril 2011].

HEREDIA HERRERA, A. Archivística general: teoría y práctica. Sevilla: Diputación Provincial, 1989.

HEREDIA HERRERA, A. "Gestión documental y calidad". VII Congreso de Archivología del Mercosur. Viña del Mar, 23-27 de Noviembre de 2007.

HEREDIA HERRERA, A. Lenguaje y vocabulario archivisticos: Algo más que un diccionario. Sevilla: Junta de Andalucía, 2011. 
JIMÉNEZ PIANO, M. "Evaluación de sedes Web". Revista Española de Documentación Cientifica, 2001, 24/4:405-431.

JOINT, N. "Designing interfaces for distributed electronic collections: the lessons of tradicional librarianship". Libri, 2001, 51:148-156.

KIM, H.; LEE, H. "Digital-age trends and perspectives in Korean university archives". The Electronic Library, 2007, 27/3: 426-440.

LODOLINI, E. Archivistica. Principios y Problemas. Madrid: Anabad, 1993.

MARTÍNEZ GONZÁLEZ, O. "El Web del Archivo General de la Universidad Carlos III de Madrid". Boletín de la ANABAD, 2000, 50/1:21-44.

MÁS BLEDA, A.; CHAÍN NAVARRO, C. "Los usuarios y los webs de los archivos históricos nacionales: el caso del Arquivo Nacional da Torre do Tombo (Portugal)". Investigación Bibliotecológica: archivonomía, bibliotecología e información, 2009, 23/47: 189-212.

MÁS BLEDA, A., SÁNCHEZ BAENA, J. J., CHAIN NAVARRO, C. "Los archivos nacionales de la América Hispana y su inmersión en Internet. Presencia, contenidos y disponibilidad informativa". Revista Nuevo Mundo, Mundos Nuevos [en línea], 2007, 7. Disponible en "http://nuevomundo .revues.org/document9633.html" [Consulta: 5 Mayo 2011].

NIELSEN, J.; THAIR, M. Usabilidad de páginas de inicio: Análisis de 50 sitios web. Madrid: Pearson Educación, 2002.

NOVLJAN, S.; ŽUMER, M. "Web pages of Slovenian public libraries: evaluation and guidelines". Journal of Documentation, 2004, 60/1:62-76.

OSORIO, N. L. "Web sites of science-engineering libraries: an analysis of content and desing". Issues in Science and Technology Librarianship [en línea], 2001. Disponible en "http://www.library.ucsb.edu/istl/01-winter/refereed.html" [Consulta: 13 Junio de 2011].

PERPINYÁ MORERA, R. "Instrumentos de selección de software para la gestión de archivos". Bilduma, 2000, 14:301-333.

PROVIDENTI, M. "Library Web Accessibility at Kentucky's 4-year Degree Granting Colleges and Universities". D-Lib Magazine [en línea], 2004, 10/9. DOI:10.1045.

Disponible

en

"http://www.dlib.org/dlib/september04/providenti/09providenti.html"

[Consulta: 10 de Junio 2011].

ROBA STUART, O. "Archivos de datos en línea para ciencias sociales". El profesional de la información, 2003, 12/5:400-410.

RODRÍGUEZ BRAVO, B. "Los repositorios de información, guardianes de la memoria digital". Anales de Documentación, 2007, 10:361-374.

RUÍZ PÉREZ, S.; MARTÍN FERNÁNDEZ, F. J.; PLASENCIA RODRÍGUEZ, E. "Evaluación de sitios Web de Archivos Nacionales e Históricos provinciales españoles". Tria: Revista de Asociación de Archiveros de Andalucía, 1999, 6: 165-174. 
SCHELlEMBERG, T. R. Archivos Modernos. Principios y Técnica. La Habana: Instituto Panamericano de Geografía e Historia, 1958.

SCHMETZKE, A. "Web Accessibility at University of Wisconsin Libraries". Newsletter [en línea], 2005, 22/3:5-9. Disponible en

"http://www.wla.lib.wi.us/waal/newsletter/223.html\#webaccess" [Consulta: 10 Junio 2011].

SIERRA ESCOBAR, L. F. "Como medir la eficiencia, eficacia y efectividad en los archivos: propuesta de indicadores de gestión". Actas de la IV Convención Internacional de Archivistas. Coindear San Bernardo (Chile), 10-13 de abril de 2012.

SPINDLER, T. "The Accessibility of Web Pages for Mid-sized College and University Libraries". Reference y User Services Quarterly, 2002, 42/2:149154.

STOVER, M.; ZINK, S. D. "World Wide Web home page desing: patterns and anomalies of higher education library home pages". Reference Services Review, 1996, 7-20.

TORRADO MORALES, S. "La página web del archivo histórico del Instituto Luce: un instrumento de difusión cinematográfica". Anales de Documentación, 2007, 10:413-428. 